\title{
Three-Dimensional Broadband Omnidirectional Acoustic Ground Cloak
}

\author{
Lucian Zigoneanu, ${ }^{1}$ Bogdan-Ioan Popa,${ }^{1}$ and Steven A. Cummer ${ }^{1, *}$ \\ ${ }^{1}$ Department of Electrical and Computer Engineering, \\ Duke University, North Carolina 27708
}


The control of sound propagation and reflection has always been the goal of engineers involved in the design of acoustic systems. A recent design approach based on coordinate transformations, which is applicable to many physical systems ${ }^{1-13,15}$, together with the explosive development of a new class of engineered materials called metamaterials, has opened the road to the unconstrained control of sound. However, the ideal material parameters prescribed by this methodology are complex and challenging to obtain experimentally, even using metamaterial design approaches. Not surprisingly, experimental demonstration of devices obtained using transformation acoustics is difficult, and has been implemented only in two-dimensional configurations ${ }^{10,16}$. Here, we demonstrate the design and experimental characterization of an almost perfect threedimensional, broadband, and, most importantly, omnidirectional acoustic device that renders a region of space three wavelengths in diameter invisible to sound.

It is well understood that, given an arbitrary geometric transformation of a sound field, the effective mass density and the bulk modulus required to implement that transformation is determined $\mathrm{as}^{5,17}: \overline{\bar{\rho}}^{r}=\operatorname{det}(A)\left(A^{-1}\right)^{T} \overline{\bar{\rho}}^{v} A^{-1}$ and $B^{r}=\operatorname{det}(A) B^{v}$, where $\overline{\bar{\rho}}$ is the mass density tensor, $B$ is the bulk modulus, $A$ is the Jacobian matrix of the transformation and $r$ and $v$ denotes the real and virtual space, respectively. One application of the coordinate transformation method that received significant attention, and that we focus on here, is the so called "ground cloak". The ground cloak is a material shell that when placed over arbitrary objects sitting on reflecting surfaces, i.e. ground, makes the object undetectable using sound radiation. The concept has been introduced in the context of electromagnetics ${ }^{18-20}$, but has rapidly been extended to other physical systems, including acoustics ${ }^{10}$.

The coordinate transformation technique enabling these cloaking devices is especially suitable for acoustics. Developed by noticing the similarity between the acoustic wave equation and the conduction equation ${ }^{5}$, the method requires a wide range of anisotropic and inhomogeneous material parameters. Unlike electromagnetics, however, these are easier to realize in acoustics in a broadband manner using metamaterial methods because conventional materials have a broad range of acoustic material parameters spanning multiple orders of magnitude.

There have been attempts to avoid the difficulties associated with the coordinate transformation approach by using more conventional techniques. However, these entail lowering 
design requirements, such as replacing omnidirectionality with unidirectionality, and have been proven very challenging as well when applied experimentally ${ }^{14,26}$. Here we show that omnidirectional three-dimensional ground cloaks obtained using coordinate transformation methods are feasible in practice.

There are several options for geometric transformations that will map the volume occupied by the object to hide into a flat region. The so-called quasi-conformal map ${ }^{8,18,20}$ results in isotropic material parameters but requires a cloaking shell many times larger than the object to be hidden. A simple unidirectional transformation ${ }^{21-24}$ results in a much more compact cloaking shell but requires strongly anisotropic effective material properties. This is the design approach we take.

The geometry and transformation are depicted in Fig. 1, where the object that we want to hide is a square pyramid placed on a reflecting surface. The entire cloaked space is split into four separate quadrants. We find a proper transformation for each of them. For example, for the region characterized by $y \geqslant|x| \geqslant 0$, i.e. the cut-out region shown in the top panel of Fig. 1, a suitable transformation that results in homogeneous material parameters is: $u=x, v=y, w=\frac{c}{c-a}\left(\frac{a}{b}|y|+z-a\right)$, where $(x, y, z)$ are the coordinates in the real space and $(u, v, w)$ are the coordinates of the virtual space. The constants $\mathrm{a}, \mathrm{b}$, and $\mathrm{c}$ are the pyramidal object dimensions given in Fig. 1, namely $a=5.7 \mathrm{~cm}$ is the object height, $c=11.4$ $\mathrm{cm}$ is the cloaked region height and $b=17.15 \mathrm{~cm}$ is half the pyramid base width.

Following the transformation procedure, the material parameters required by the ideal cloak are $\rho_{11}^{p r}=2.28, \rho_{22}^{p r}=0.5, \rho_{33}^{p r}=0.44$, and $B^{p r}=0.5$, where $p r$ denotes the principal components (eigenvalues) of the mass density tensor and bulk modulus. The orientation of the principal axes relative to the original coordinate system $x y z$ is the same inside each quadrant, but varies from quadrant to quadrant. For example, for the same region that has the transformation specified above, the principal axis labeled 3 is parallel to the $O x$ axis, principal axis 2 is in the $y O z$ plane and makes a $23^{\circ}$ angle with the $O y$ axis. Principal axis 1 is perpendicular on the plane formed by 2 and 3 . These material parameters are relative to the background material parameters, which is air in our case. A material that is less dense and more compressible than air is difficult to obtain with passive acoustic metamaterials.

However, if we begin the design procedure with a pyramidal object in a virtual space that has relative (to air) density and bulk modulus of $m$ (called scaling factor) times higher than for air, then we find the cloaking shell parameters are $\rho_{11}^{p r, n e w}=m \rho_{11}^{p r}, \rho_{22}^{p r, n e w}=m \rho_{22}^{p r}$, 


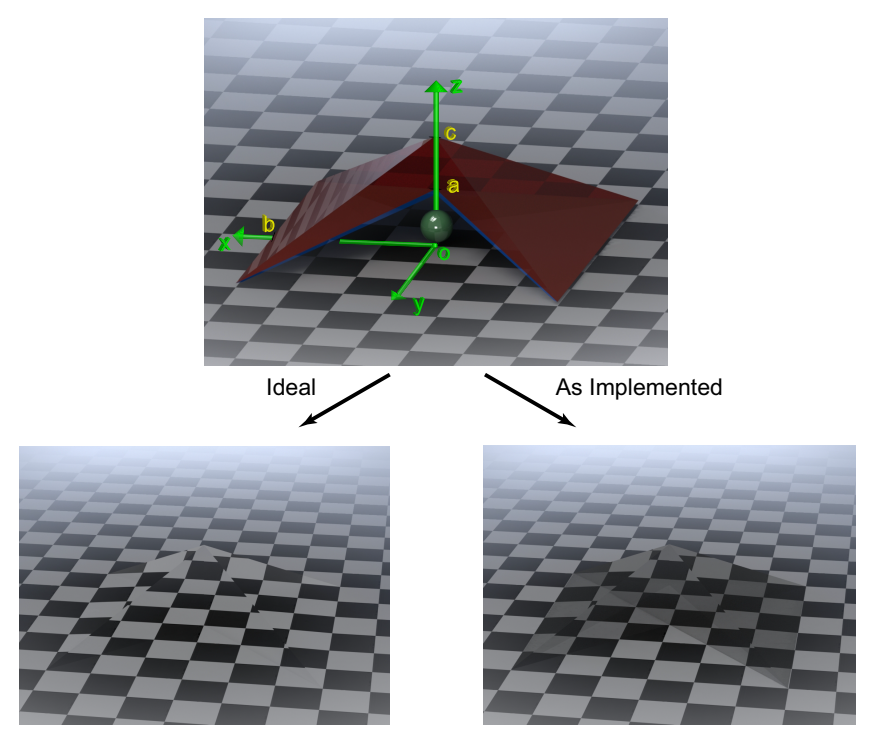

FIG. 1: Schematic representation of the pyramid shaped acoustic ground cloak. The cloak (blue region) was designed to cover, at $3 \mathrm{kHz}$, a hollow square pyramid (blue thin sheet) 34.3 $\mathrm{cm}(3 \lambda)$ long and $5.7 \mathrm{~cm}$ tall $(0.5 \lambda)$, placed on the ground. $\lambda$ is the wavelength in air at the design frequency. The cloak shares the same base with the object we wanted to hide and extends in the vertical direction from $5.7 \mathrm{~cm}(0.5 \lambda)$ to $11.4 \mathrm{~cm}(\lambda)$. One quarter of the cloak is removed in order to highlight that any object (e.g. the green sphere) could be placed inside. The bottom panels qualitatively illustrate the expected performance of the ground cloak with the ideal (perfect) and fabricated (imperfect but good) material parameters.

$\rho_{33}^{p r, n e w}=m \rho_{33}^{p r}$, and $B^{p r, n e w}=m B^{p r}$. For $m=2.5$, all the material parameters required by the cloaking can be realized using passive acoustic metamaterials. This change has the effect of making the cloaked object behave like a pyramid of relative density and bulk modulus of 2.5 instead of air (as depicted in Figure 1). This is an object with the same sound velocity as air but with a modest impedance mismatch and thus small reflection at the interface with air ${ }^{10}$. Numerical simulations, shown in the Supplemental Material - Section II, were performed and revealed that indeed the implemented cloak produces a minimal scattering.

For this design, two principal components of the mass density tensor have close values, thus a similar unit cell as for the two-dimensional cloak reported in Ref. [10] can be used. The unit cell is a $5 \mathrm{~mm}$ air filled cube which has in the middle an acrylic perforated plate of $1.6 \mathrm{~mm}$ thickness and $0.85 \mathrm{~mm}$ hole radius. These dimensions determine the mass density 


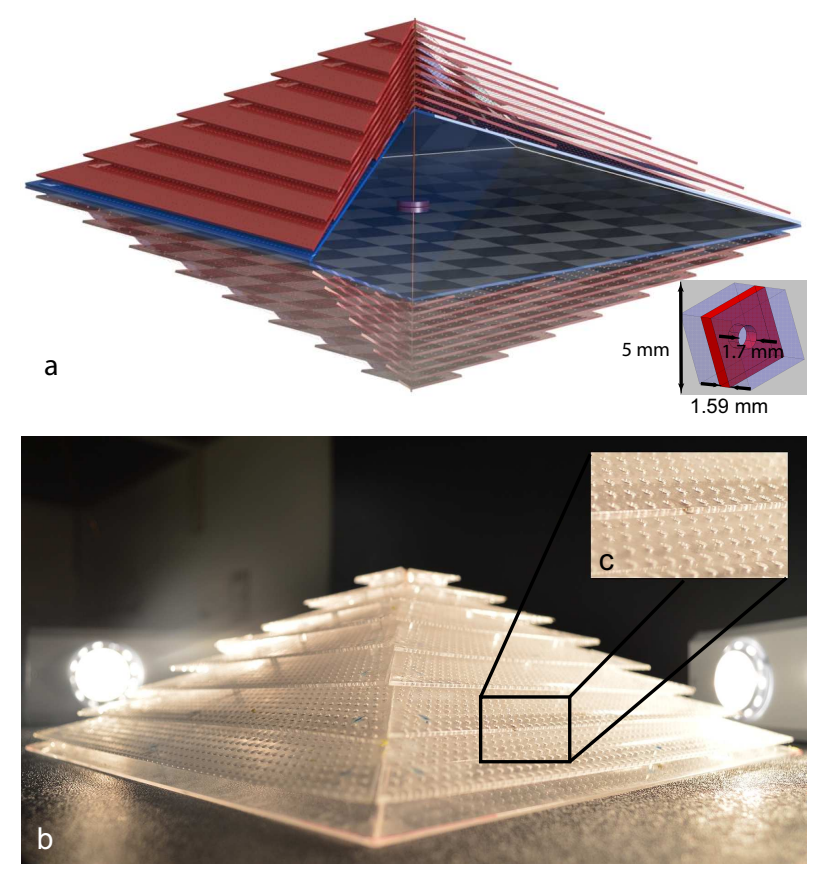

FIG. 2: Snapshots of the fabricated cloak. a, Physical structure of the designed cloak and unit cell dimensions. One quarter is not shown in order to highlight the cross sectional view and the holding support; $\mathbf{b}$, Photograph of the actual cloaked object, placed on the ground. c, Details of the perforated plates. The perforations are made using a laser cutting machine and clear cast acrylic sheets.

components (the higher value for the wave propagating perpendicular to the plate and the lower values for the wave propagating parallel to the plate). The unit cell is about 23 times smaller than the wavelength at the design frequency, $3 \mathrm{kHz}$; therefore, the homogenization theory holds. Further investigations of this unit cell behavior ${ }^{10}$ showed that the acoustic transmission losses are modest and its effective material parameters (mass density and bulk modulus) are relatively constant for frequencies up to $6 \mathrm{kHz}$. The metamaterial structure obtained by replicating these unit cells is a medium of parallel perforated plates. The metamaterial principal axis is determined for each region of the cloak using the same procedure as described in Ref. [10]. The principal axis direction imposed the angle of $23^{\circ}$ made by the perforated plates with the horizontal plane. All the perforated plates were made using a universal laser cutting machine and clear cast acrylic sheets. Other solids with the same dimensions could be used for this design, with similar results due to the high impedance 
contrast between them and the air background.

The resulting cloak covering the object is presented in Fig. 2. As can be seen, the object that we want to hide is hollow and consists only of one layer of plain (i.e. not perforated) acrylic sheet. There is virtually no difference between a solid pyramid and a hollow pyramid. This was confirmed by other experiments (not shown here) performed in a 1D acoustic waveguide and which compared the reflection of several "perfect acoustic reflectors" (e.g. multiple layers of acrylic sheets, thin/thick aluminum plates, multiple types of woods, etc.). We performed numerical simulations to confirm that the inherent fabrication errors (e.g. larger/smaller holes than desired or slightly different unit cell dimensions due to errors in positioning the perforated plates) do keep the effective material parameters close to the desired values (i.e. errors smaller than $20 \%$ ).

To evaluate the performance of our device, we constructed the experimental setup presented in Fig. 3. An almost omnidirectional sound source was set to send a short, therefore broadband, sound pulse towards the tip of the pyramidal object and perpendicularly towards the ground. This illumination was chosen to test the absolute performance of the cloak, as the incident wave hits the most uneven part of the object and cloaking device. The object and cloaking device have sharp edges in this region, and the cloaking metamaterial has discontinuities in the direction of the material principal axis, therefore the scattering is maximized in this configuration. As we will see next, even for this unfavorable wave incidence, the cloaking shell performs almost perfectly.

Three cases were measured: with nothing on the ground (the ideal case), with only the object on the ground, and with the cloaked object on the ground, respectively. The incident sound $^{25}$ is a short Gaussian pulse of $600 \mu$ s half amplitude duration modulated with a 3 $\mathrm{kHz}$ sinusoidal, i.e. the pulse half amplitude duration is slightly less than two periods. The bandwidth of this pulse, defined as the ratio between the half amplitude band to the center frequency of $3 \mathrm{kHz}$, is $42 \%$. Two microphones were used to measure the acoustic wave propagation. The first one was placed in a fixed position and was used as a time reference while the other one scanned a specific region above the cloak. The planar area in which the sound is measured in this setup is shown in Fig. 3 together with an actual measurement of the sound produced by the source. At every measurement location, several measurements are performed and averaged in order to increase the signal-to-noise ratio. As can be seen from the figure, the source is quasi-omnidirectional and sends waves characterized by wavevectors 

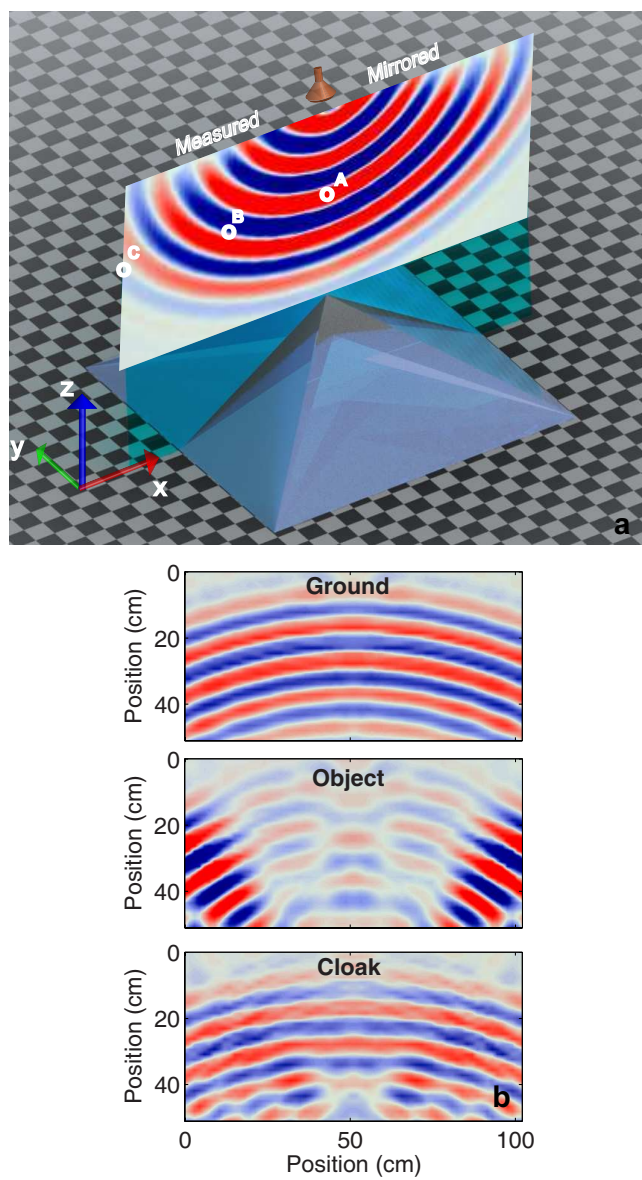

FIG. 3: Experimental setup and qualitative results. a, Experimental setup schematic diagram. The scanned area is $50 \mathrm{~cm}$ wide and $50 \mathrm{~cm}$ tall. This region was selected such that the cloaking effect could be clearly identified (i.e. the attenuation due to free space travel of the wave is minimal). The scanning microphone is moving on a square grid that ensures at least 4 measurement points per wavelength, in both directions. A, B, C are three particular measurement locations. b, Instantaneous scattered pressure field snapshot which highlights the object alone acoustic signature and how the cloaked object mimics the empty ground. These plots contain the measured region and a mirrored region, in order to cover a larger section. A motion picture showing the incident and scattered pressure fields is presented in the Supplementary Movie S1.

spanning a 28 degree sector that contains the object as seen from the source. The goal of this experimental setup was to qualitatively and quantitatively demonstrate that the acoustic signature of the object alone is significantly minimized when it is covered with the cloak.

The instantaneous reflected pressure field for the three cases are shown in Fig. 3. As 

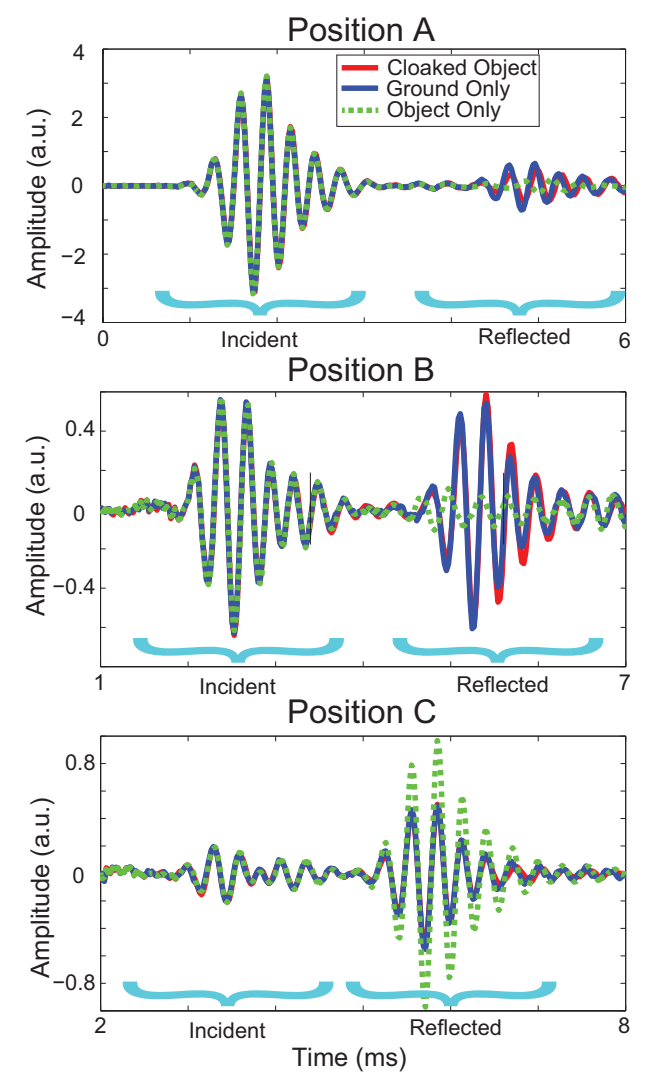

FIG. 4: Measured signals at three different locations A, B, C. The exact location of these examples is specified in Fig. 3 a. Each plot shows the incident and scattered acoustic pressure for three scenarios: empty ground, object placed on the ground and cloaked object placed on the ground.

already mentioned, the direction of the incident sound pulse was chosen so that the pulse impinges on the tip of the pyramidal object and cloaking shell, in the region with most sharp edges, where the cloaking device has discontinuities in the material principal axis. This is the situation which produces the most scattering and, therefore, the case in which the object is most visible. Figure 3 (b) confirms this analysis. The top panel shows the expected specular reflection off the bare ground in the form of a spherical wavefront. In contrast, the object significantly disturbs this spherical field distribution. Its sharp edges generate discontinuities in the reflected wave phase profile at the horizontal positions of 25 $\mathrm{cm}, 50 \mathrm{~cm}$ and $75 \mathrm{~cm}$, as well as reduced backscattering towards the source and, in the same time, enhancement of the reflected field in the directions that correspond to specular reflections from the object flat faces. 
Remarkably, the cloaked object reflects nearly identical to the flat reflecting plane [see the bottom panel in Fig. 3 (b)]. Thus, the reflected phase front returns to the uniform spherical shape as if no object is placed in between the source and ground. The wave amplitude distribution is almost identically recovered as well, except for modest attenuation that we attribute to losses in the perforated sheets used to construct the shell. A notable aspect of this experimental result is that it agrees very well with the theoretical predictions given by the transformation acoustics theory, and, yet, it is counter-intuitive in that sharp edges and material parameter discontinuities in the metamaterial structure allow an almost perfect recovery of the ideal fields scattered by the bare ground. An animation of the instantaneous wave propagation emphasizes this result and is available in the Supplementary Movie S1. In order to thoroughly confirm the cloaking effect, several other additional scanning planes and angles of incidence have been tested. The description of the experimental setups and the corresponding results (i.e. instantaneous measured pressure fields and matching movies) are presented in detail in the Supplemental Material - Section III and Supplementary Movies S2 through S6.

In order to quantitatively show the cloak performance, we accompany the previous experimental results with a set of individual time domain measurements (see Fig. 4) taken at specific points, labeled A, B, and C in Fig. 3(a). The time domain sound wave recorded at position A (see the top panel of Fig. 4) corresponds to the backscattering direction. The plot shows the incident short Gaussian pulse, together with the reflection from the ground reflector, bare object, and cloaked object. As expected, the incident pulses are identical in all three cases. Remarkably, the phase of the wave reflected by the cloaking device is within $15^{\circ}$ of the ideal phase, while the amplitude is $74 \%$ of the ideal amplitude, in stark contrast with the difference between the wave reflected by the object and the ideal wave form (phase difference of approximately $-90^{\circ}$, and an amplitude ratio of $33 \%$ ). Position $\mathrm{B}$ corresponds to the direction in which the wave reflected by the bare object is minimum, while position $\mathrm{C}$ corresponds to the direction in which the specular reflection from the object faces are maximum. In both cases, the cloaking shell almost perfectly corrects the pulse phase advance and, at the same time, brings the amplitude up or down to match it with the ideal fields.

The designed and fabricated 3D reflecting plane cloak performs well and demonstrates that the design methodology used is a robust one. These experimental results provide a clear path for realizing three-dimensional acoustic devices for the manipulation of sound 
reflection. They also show that complex three-dimensional transformation devices can be implemented using current technologies. Given the already reported new improvements in the achievable acoustic material parameters using active metamaterial structures, we expect that the range of realizable transformation acoustics devices will improve dramatically in the near future.

\section{Acknowledgements}

This work was supported by Multidisciplinary University Research Initiative grants from the Office of Naval Research (N00014-13-1-0631) and from the Army Research Office (W911NF09-1-00539).

\section{Author contributions}

B-I.P. and L.Z. performed the simulations. L.Z. conducted the fabrication, experimental design and measurements. All three authors equally contributed to the development of the project and to the text of the paper.

\section{Additional information}

Supplementary information is available in the online version of the paper. Reprints and permissions information is available online at www.nature.com/reprints. Correspondence and requests for materials should be addressed to S.A.C.

\section{Competing financial interests}

The authors declare no competing financial interests.

* Electronic address: cummer@ee.duke.edu

1 J. B. Pendry, D. Schurig, and D. R. Smith, Science 312, 1780 (2006).

2 U. Leonhardt, Science 312, 1777 (2006).

3 D. Schurig, J. J. Mock, B. J. Justice, S. A. Cummer, J. B. Pendry, A. F. Starr, and D. R. Smith, Science 314, 977 (2006).

4 S. A. Cummer and D. Schurig, New Journal of Physics 9, 45 (2007).

5 H. Y. Chen and C. T. Chan, Applied Physics Letters 91, 183518 (2007).

6 W. Cai, U. K. Chettiar, A. V. Kildishev, and V. M. Shalaev, Nat Photon 1, 224 (2007).

7 M. Farhat, S. Guenneau, and S. Enoch, Phys. Rev. Lett. 103, 024301 (2009).

8 T. Ergin, N. Stenger, P. Brenner, J. B. Pendry, and M. Wegener, Science 328, 337 (2010). 
9 X. Chen, Y. Luo, J. Zhang, K. Jiang, J. B. Pendry, and S. Zhang, Nature Commun 2, 176 (2011).

10 B.-I. Popa, L. Zigoneanu, and S. A. Cummer, Phys. Rev. Lett. 106, 253901 (2011).

11 N. Stenger, M. Wilhelm, and M. Wegener, Phys. Rev. Lett. 108, 014301 (2012).

12 S. Narayana and Y. Sato, Phys. Rev. Lett. 108, 214303 (2012).

13 N. Landy and D. R. Smith, Nat Mater 12, 25 (2013).

14 L. Sanchis, V. M. Garcia Chocano, R. Llopis Pontiveros, A. Climente, J. Martinez Pastor, F. Cervera, and J. Sanchez Dehesa, Phys. Rev. Lett. 110, 124301 (2013).

15 R. Schittny, M. Kadic, S. Guenneau, and M. Wegener, Phys. Rev. Lett. 110, 195901 (2013).

16 S. Zhang, C. Xia, and N. Fang, Physical Review Letters 106, 024301 (2011).

17 S. A. Cummer, M. Rahm, and D. Schurig, New Journal of Physics 10, 115025 (2008).

18 J. Li and J. B. Pendry, Phys. Rev. Lett. 101, 203901 (2008).

19 R. Liu, C. Ji, J. J. Mock, J. Y. Chin, T. J. Cui, and D. R. Smith, Science 323, 366 (2009).

20 J. Valentine, J. Li, T. Zentgraf, G. Bartal, and X. Zhang, Nat Mater 8, 568 (2009).

21 W. Li, J. Guan, Z. Sun, W. Wang, and Q. Zhang, Opt. Express 17, 23410 (2009).

22 J. Zhang, Y. Luo, and N. A. Mortensen, Opt. Express 18, 3864 (2010).

23 Z. Weiren, D. Changlin, and Z. Xiaopeng, Applied Physics Letters 97, 131902 (2010).

24 B.-I. Popa and S. A. Cummer, Phys. Rev. B 83, 224304 (2011).

25 L. Zigoneanu, B. I. Popa, A. Starr, and S. A. Cummer, Journal of Applied Physics 109, 054906 (2011).

26 V. M. Garcia-Chocano, L. Sanchis, A. Diaz-Rubio, J. Martinez-Pastor, F. Cervera, R. LlopisPontiveros, and J. Sanchez-Dehesa, Applied Physics Letters 99 (2011).

27 H. F. Ma and T. J. Cui, Nature Communications 1, 21 (2010). 


\section{D Broadband Omnidirectional Acoustic Ground Cloak - Supplemental Material}

This document describes the detailed design procedure for the 3D acoustic ground cloak, the simulations predicting the good performance of the implemented carpet cloak and the measurements performed to experimentally confirm the $3 \mathrm{D}$ transformation acoustics device.

\section{DESIGN METHOD FOR THE SQUARE PYRAMID CLOAK}

1. We consider the particular case of a square based pyramid cloak presented in Fig. 1. As can be seen, the entire volume is split into four regions (quadrants). For example, the quadrant 1 corresponds to the region where $x \geqslant|y| \geqslant 0$ and the quadrant 2 corresponds to the region where $y \geqslant|x| \geqslant 0$.

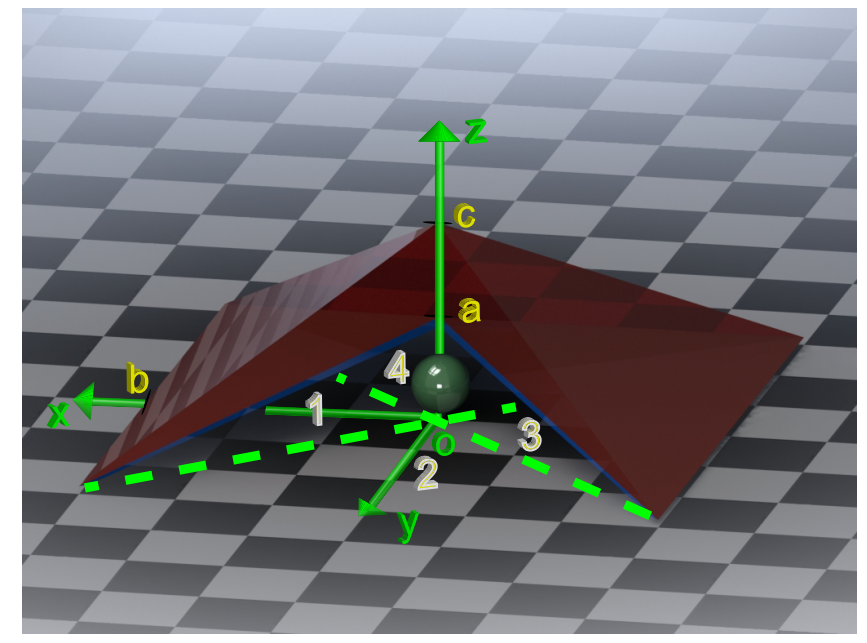

FIG. 1: Schematic representation of the square pyramid cloak and its orientation.

A suitable transformation that will meet the requirements presented in the manuscript is for quadrants 1 and 3:

$$
\left\{\begin{array}{l}
u=x \\
v=y \\
w=\frac{c}{c-a}\left(\frac{a}{b} \operatorname{sign}(x) x+z-a\right),
\end{array}\right.
$$

and for quadrants 2 and 4 :

$$
\left\{\begin{array}{l}
u=x \\
v=y \\
w=\frac{c}{c-a}\left(\frac{a}{b} \operatorname{sign}(y) y+z-a\right)
\end{array}\right.
$$

where $(x, y, z)$ are the coordinates in the real space, $(u, v, w)$ are the coordinates of the virtual space and $a=5.7$ $\mathrm{cm}, c=11.4 \mathrm{~cm}$ and $b=17.15 \mathrm{~cm}$ are the pyramids dimensions.

2. After the transformation is found, the $\overline{\bar{\rho}}^{r}=\operatorname{det}(A)\left(A^{-1}\right)^{T} \overline{\bar{\rho}}^{v} A^{-1}$ and $B^{r}=\operatorname{det}(A) B^{v}$ equations are used to find the ideal material parameters (i.e. bulk modulus and density) of the cloak. Then, these parameters are scaled up by $m$ factor (2.5 in our case) to find the practical material parameters.

3. Using again the relations from above, three angles of rotation of the original coordinate system are determined in order to transform the mass density tensor in a diagonal one. More specifically, if $R$ is the general 3D rotation 
matrix, then $\overline{\bar{\rho}}^{d}=\operatorname{det}(R)\left(R^{-1}\right)^{T} \overline{\bar{\rho}}^{r} R^{-1}$ and $B^{r}=\operatorname{det}(R) B^{v}$, where $\rho^{r}$ is the mass density tensor from the previous step and $\rho^{d}$ is the diagonal mass density tensor (i.e. a matrix that has the eigenvalues of the original mass density tensor on its diagonal and all the other elements are zero). This equation is used to determine the three angles of rotation that are in the matrix $R$. For example, for the quadrant 1 , these angles are $90^{\circ}, 113^{\circ}$, and $180^{\circ}$, respectively.

4. Three sets of numerical simulations are performed in order to design the thickness and the hole diameter of the perforated plates. The complete details of these simulations are presented in Ref. [1]. The unit cell that we chose to produce the desired material parameters is a $5 \mathrm{~mm}$ air filled cube in which we placed in the middle a $1.5875 \mathrm{~mm}$ thick acrylic sheet with a circular perforation of $1.7 \mathrm{~mm}$ diameter.

5. Multiplying the unit cell in the three directions will produce a medium of parallel perforated plates. In our particular case, the distance between two consecutive plates is $5 \mathrm{~mm}$ and the orientation of these plates is given by the orientation of each unit cell. In order to populate the cloak with perforated plates, we start from the tip of the small pyramid (i.e. the object that we want to hide) and measure the 1.762 mm (that is the air gap up to the first perforated plate) and go up through the central supporting pole and place the plates according with the required orientation at every $5 \mathrm{~mm}$. More specifically, each quadrant will have 11 perforated plates. In order to create the triangular perforated plates, we start with a proper solid plate. Then, we start from a corner and create an array of holes with $1.7 \mathrm{~mm}$ diameter and equally placed in both directions at every $5 \mathrm{~mm}$. The holes that are too close to the edge of the plate will be eliminated. This procedure will lead to triangular perforated plates, 11 for each quadrant (i.e. 44 in total). We should note also that close to the edges of the regions we will have incomplete unit cells.

6. Every plate is individually cut using a universal laser cutting machine and then placed at its location using properly designed holders and spacers.

\section{THEORETICAL COMPARISON BETWEEN IDEAL AND IMPLEMENTED CLOAK PERFORMANCE}

Numerical simulations were performed to emphasize the relatively small pressure field disturbance created but the not ideal material parameters of the cloak. These results are presented in Fig. 2 and they show the scattered field for a perfect impedance match between the cloaking pyramid and the air background and for the case when there is a small mismatch between the two impedances (i.e. for our experimental realization). We also show the pressure field maps for the object only placed on the ground, in order to emphasize how its acoustic signature is erased almost completely by our cloaking device.

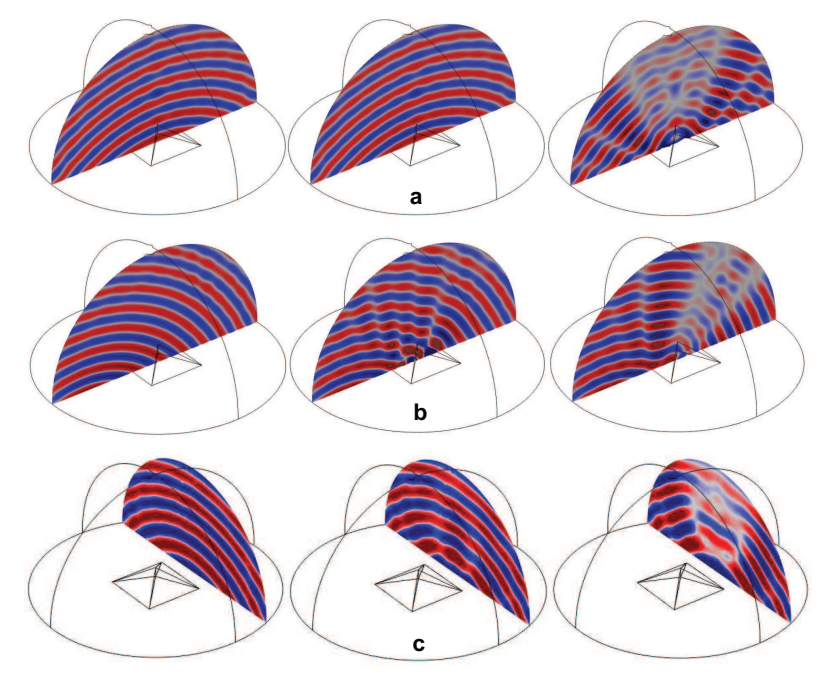

FIG. 2: Simulated scattered pressure field for the ideal cloak (left column), the experimental cloak (middle column) and object (right column) for three particular cases: a) source placed on top, b) source placed at $45^{\circ}$, plane $x O z$ and c) source placed at $45^{\circ}$, plane $y O z, x=25 \mathrm{~cm}$. 


\section{EXPERIMENTAL MEASUREMENTS PERFORMED TO CONFIRM THE OMNIDIRECTIONAL CLOAKING EFFECT}

In this section we describe the supplementary experimental setups that were used to scan the pressure field maps in three situations (ground only, object placed on the ground and cloaked object placed on the ground).

1. In addition to the scanned pressure field map presented in the manuscript, the top incidence performance was tested in a different plane, as shown in Fig. 3. The dimensions of this scanning plane are the same with the one in the manuscript, $50 \mathrm{~cm} \times 50 \mathrm{~cm}$. Although the object acoustic signature is not as strong as for the plane $x O z$, the cloaking effect is clearly visible. The corresponding movie is presented as supplemental materials Movie S2.
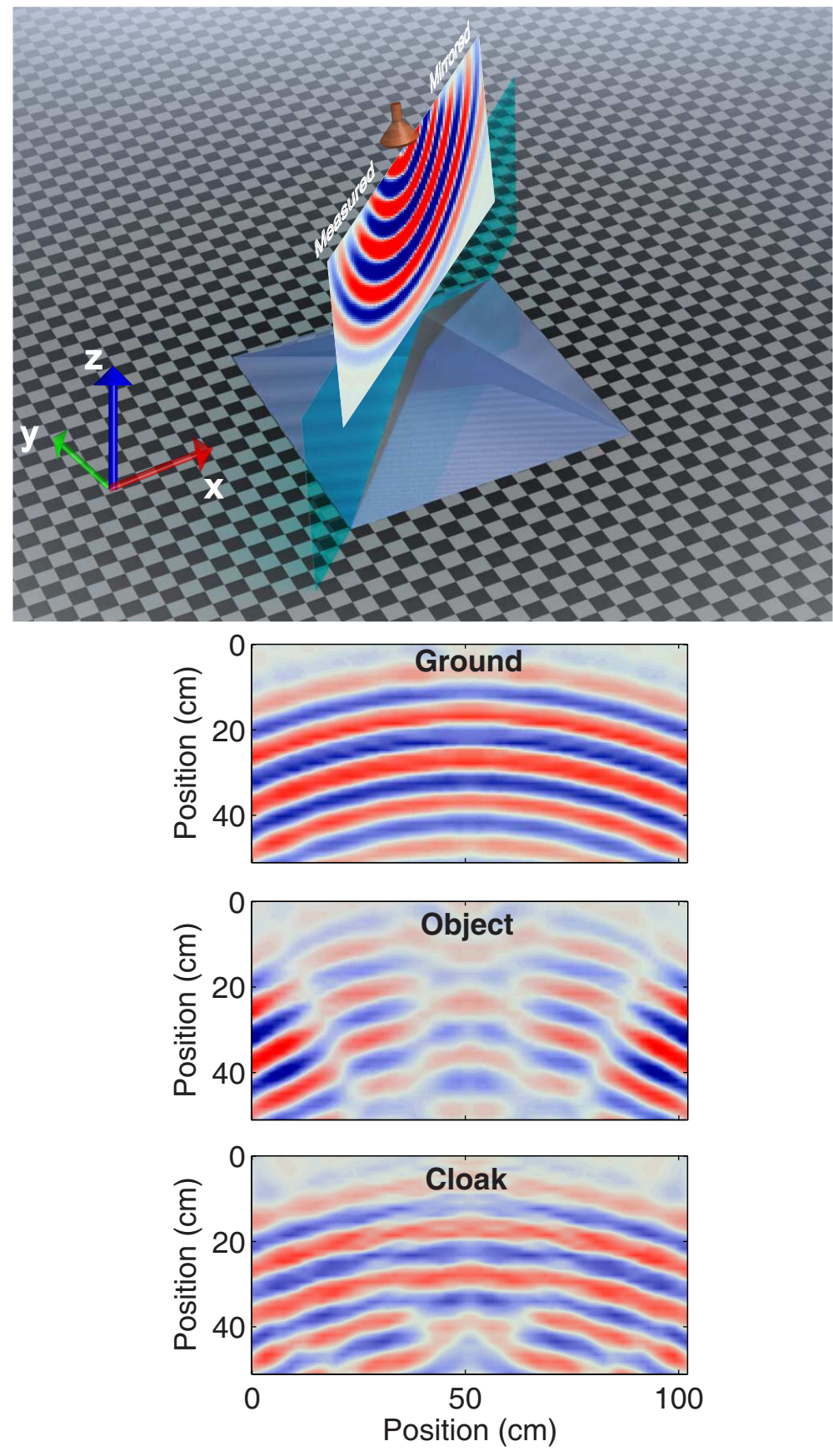

FIG. 3: Schematic representation of the additional scanning plane for the case when the sound source is placed on top. 
2. Two additional planes were scanned when the source is placed at $45^{\circ}$ and $60^{\circ}$ from the normal, like in Fig. 4 and Fig. 5. The source is at $40 \mathrm{~cm}$ in $x$ direction and at $40 \mathrm{~cm}$ in $z$ direction for $45^{\circ}$ and at $23.4 \mathrm{~cm}$ for $60^{\circ}$ ( $y=0$ for both cases).
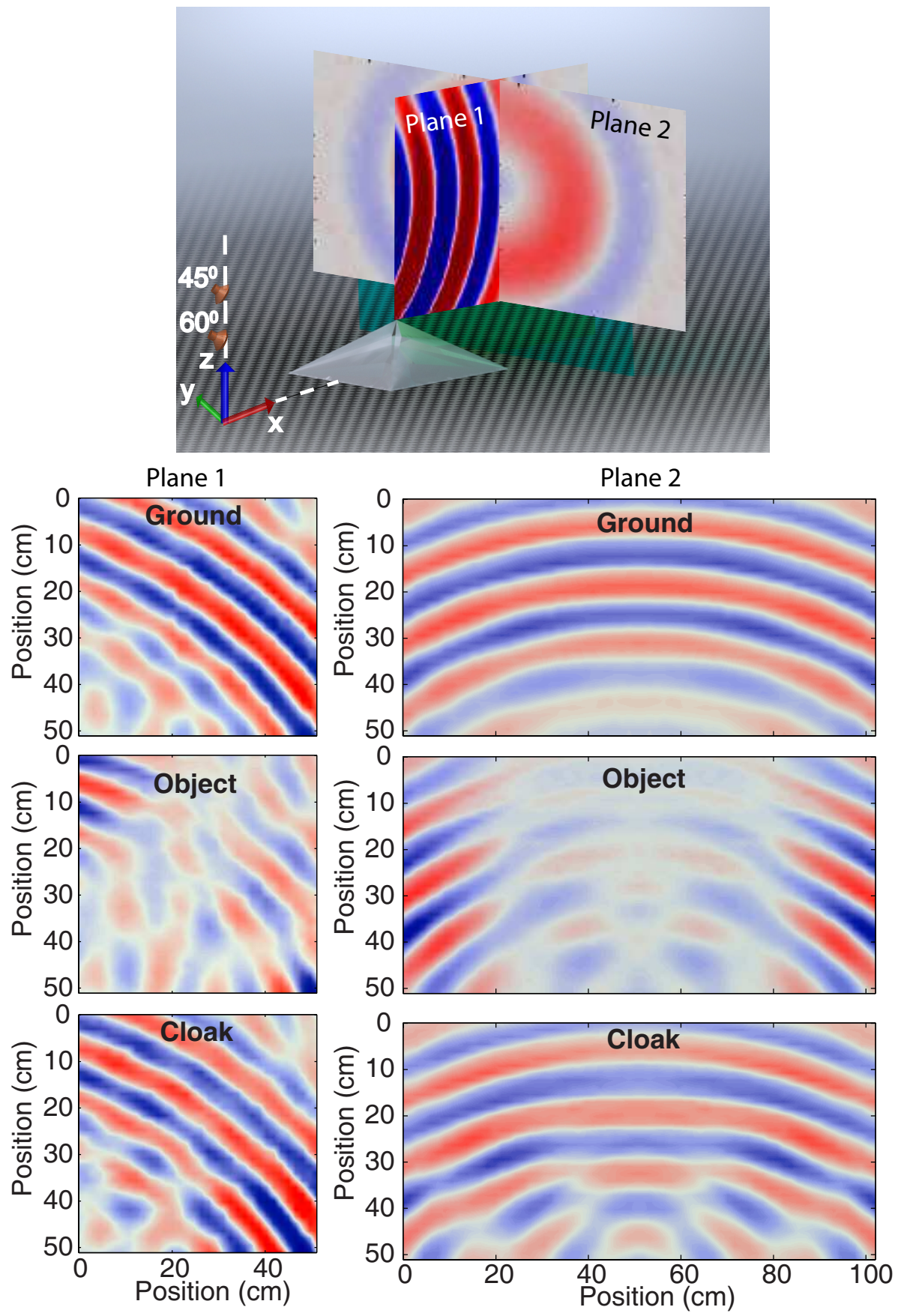

FIG. 4: Schematic representation of the scanning planes for the sound source placed at $45^{\circ}$ and the corresponding measured scattered pressure fields.

The measurements for the two planes (Plane 1 and Plane 2, placed at $x=25 \mathrm{~cm}$ away from the base center of the pyramid) include only measured data for Plane 1 and measured data and mirrored data for Plane 2. They were performed again for the three particular situations: bare ground, object placed on the ground and cloaked object placed on the ground. The corresponding movies of the propagating acoustic wave are presented in the supplemental material as Movie S3 and Movie S4 for the source placed $45^{\circ}$, and Movie S5 and Movie S6 for the case when the source is placed at $60^{\circ}$, respectively. In all the cases, the acoustic signature of the object only 
placed on the ground is clearly shown and it is significantly diminished when the object is covered with our cloaking device.
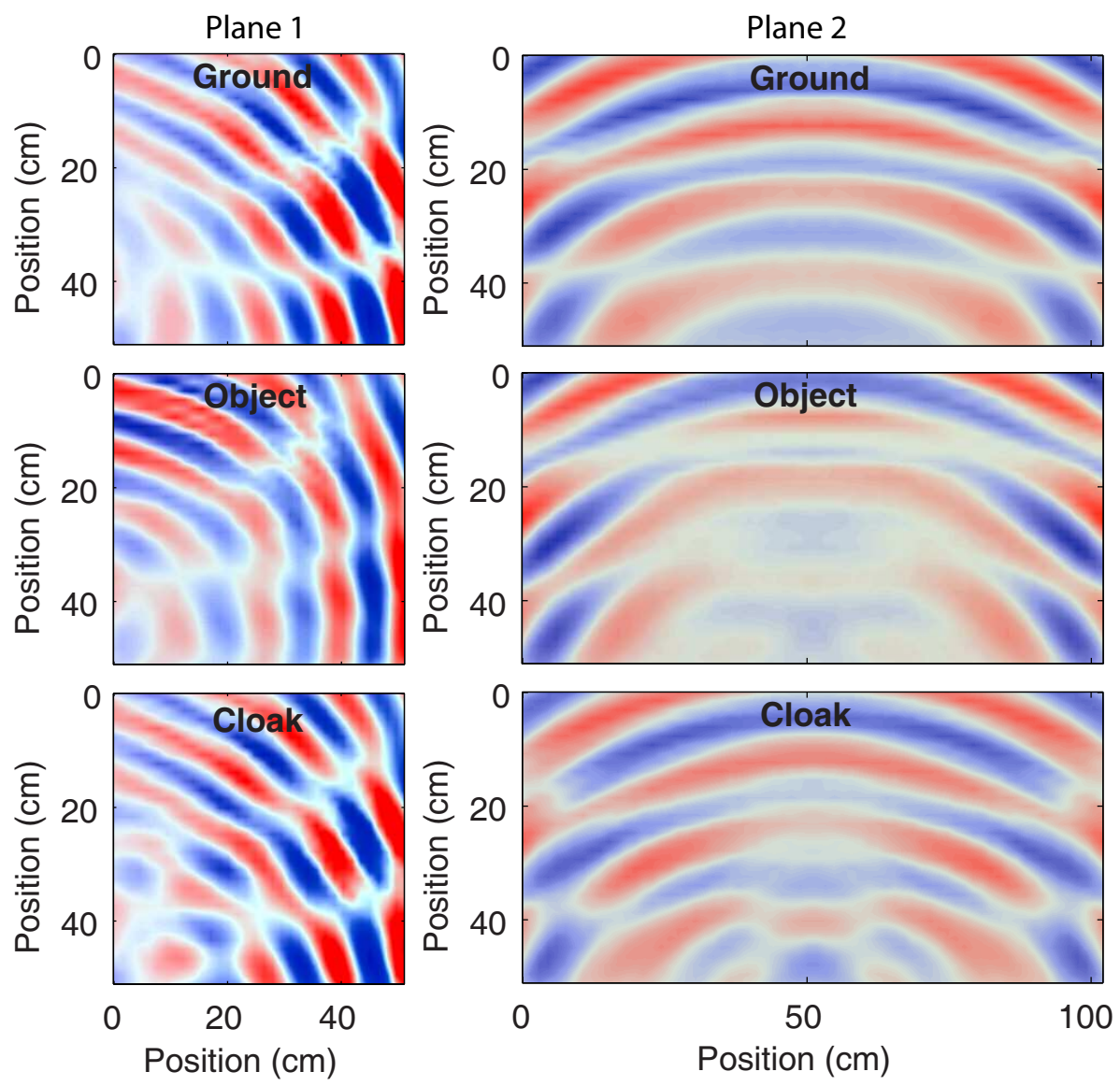

FIG. 5: The measured scattered pressure fields when the sound source is placed at $60^{\circ}$. The measured planes are exactly like in the previous case, the only difference is the source location.

[1] B.-I. Popa, L. Zigoneanu, and S. A. Cummer, Phys. Rev. Lett. 106, 253901 (2011). 